\title{
EVALUACIÓN DE PULPA DE TOTUMO (Crescentia cujete L) ENSILADA EN DOS ESTADOS DE MADURACIÓN COMO ALTERNATIVA EN ALIMENTACIÓN BOVINA
}

\author{
EVALUATION OF PULP OF TOTUMO (Crescentia \\ cujete L) ENSILADA IN TWO STATES OF MADURATION LIKE \\ ALTERNATIVE IN NUTRITION BOVINA
}

\author{
Edwin J Flórez ${ }^{1}$ \\ Recibido para publicación: Febrero 15 de 2012 - Aceptado para publicación: Mayo 23 de 2012
}

\begin{abstract}
RESUMEN
Los bovinos producidos en el Caribe colombiano durante la época de sequia presentan pérdidas de peso debido a la escasez de alimentos ricos en proteínas y carbohidratos. Con el fin de contribuir en la solución se planteó evaluar la pulpa ensilada de totumo como alternativa en la alimentación animal mediante técnicas de secado de la pulpa para reducir la concentración de ácido cianhídrico. El estudio se realizó en el Centro de Investigación y desarrollo de Ingeniería de la Universidad Popular del Cesar, Valledupar-Colombia. La pulpa se evaluó en dos estados de maduración (verde y maduro) ensilada por cuatro y ocho días, estrujada, secada al sol y al horno. Se aplicó un diseño completamente al azar de seis tratamientos y tres repeticiones. Se utilizaron métodos manuales para el despulpado. En el análisis fisicoquímico de la pulpa se usaron técnicas de titulación, refractometría, potenciometría, fotometría de Ilama, secado en mufla e hidrólisis enzimática. Los rendimientos en pulpa del totumo maduro y verde fueron de $57,87 \%$ y $59,63 \%$ respectivamente. La pulpa de totumo maduro, ensilada por cuatro días, estrujada y secada al sol, arrojó mayor porcentaje de carbohidratos con 42,71 y la de totumo verde, estrujada, sin ensilado y sin secado, fue menor con 5,44. Desde el punto de vista nutricional y de contenido de ácido cianhídrico HCN, la pulpa del totumo maduro, con ocho días de ensilado, estrujada y secada al horno se considera como la alternativa más conveniente para alimentación bovina.
\end{abstract}

Palabras clave: totumo, pulpa, ácido cianhídrico, ensilado, secado.

\begin{abstract}
Cattle produced in the Colombian Caribbean during the dry season have lost weight because of the scarcity of food rich in protein and carbohydrates. To help in the solution, the pulp silage of totumo was evaluated as an alternatively in animal feeding. Drying techniques to reduce the concentration of hydrocyanic acid of the pulp were used. The study was conducted at the Center for Research and Development of Engineering Popular University of Cesar, Valledupar, Colombia. The pulp was evaluated in two stages of maturity (green and ripe),
\end{abstract}

'Ingeniero agroindustrial, magister en ciencia y tecnología de alimentos, docente de la Universidad Popular del Cesar. Valledupar. Email: eflorezavendano@yahoo.com 
silaged during four and eight days, crushed, sun-dried and baked. A completely randomized design with six treatments and three replications was used. Manual methods were used for pulping. For physicochemical analysis of the pulp, titulation, refractometry, potentiometry, flame photometry, drying flask and enzymatic hydrolysis techniques were used. Pulp yields in mature and green totumo were $57.87 \%$ and $59.63 \%$ respectively. Totumo pulp ripe silage during four days, crushed and dried in the sun, threw a higher percentage of carbohydrates with 42.71, and the green calabash, crushed, without silage and without drying, was lower with 5.44. From a nutritional standpoint and $\mathrm{HCN}$ hydrocyanic acid content, the pulp of ripe calabash, with eight days of silage, crushed and dried in the oven is considered to be the most suitable for cattle feed.

Key words: totumo, pulp, hydrogen cyanide, silage, dry.

\section{INTRODUCCIÓN}

El árbol de totumo (Crescentia cujete L) pertenece a la familia Bignoniáceas, recibe los nombres de calabaza, jícaro, morro, tapara, estelí y guira, tiene como nombre científico Crescentia y la especie que se estudió fue cujete; éste se reproduce de manera silvestre en Centroamérica y Suramérica, se reproduce por semilla y esquejes, Ilega a tener una altura de ocho metros, un diámetro de 25 centímetros, una producción máxima a partir del octavo año de 27 kilogramos fruto por árbol / año; el fruto demora en el árbol de 5 a 7 meses antes de caer, es resistente a la sequía y a pesar de su buen contenido de proteína y carbohidratos, actualmente no se aprovecha (Zamora et al 2001).

Existe en el departamento del Cesar la necesidad de tener al alcance alimentos naturales ricos en nutrientes, que garanticen en gran parte del año la productividad y los bajos costos de producción, en los hatos ganaderos. En nuestra naturaleza, para la época de verano se encuentran alternativas de alimentación para bovinos como es el totumo, fruto silvestre cuya pulpa se usa como expectorantes, antinflatorio, laxante, purgante, calmantes de dolores menstruales, jarabes y su corteza para elaborar artesanías, cantimploras, cucharas, totumas, vasijas (Murgueitio y Ibrahim 2004).
La pulpa de totumo en sus características fisicoquímicas y nutricionales, puede facilitársela a los ganaderos como otra alternativa de alimento del ganado, ya que tradicionalmente los alimentan con pastos naturales, los cuales en épocas de sequía escasean y su calidad nutritiva (proteína, carbohidratos y fibra) se ve reducida drásticamente, convirtiéndose en un problema hasta el punto que se refleja en la baja producción y calidad de la leche y carne. Los empresarios en el afán de buscar cómo alimentar sus bovinos, compran alimentos concentrados de altos precios, lo que ha incrementado los costos de producción en la ganadería, como también en los alimentos para el consumo humano (Murgueitio y Ibrahim 2004). Considerando lo antes anotado el objetivo de la investigación es evaluar la pulpa de totumo ensilada en dos estados de maduración (totumo verde - M1 y totumo maduro - M2) como alternativa en al alimentación bovina.

\section{MATERIALES Y MÉTODOS}

Este trabajo de investigación se desarrolló entre los meses de enero a diciembre de 2010, en la planta piloto y en el Centro de Investigación y Desarrollo de la Ingeniería de la Universidad Popular del Cesar. Los totumos se cosecharon en dos estados de maduración, verde y maduro con cuatro y seis meses de proceso 
de maduración fisiológica respectivamente, despulpado con un objeto corto punzante y estrujado manual, con posterior ensilado por cuatro y ocho días en tanques de plástico hermético, a los que se les realizaron monitoreo diarios de la temperatura. Se aplicó un diseño completamente al azar con seis tratamientos y tres repeticiones; los datos fueron sometidos a análisis de varianza y a comparación de medias por Tuckey con un nivel de confianza de 95\%.

Los tratamientos fueron:

$\mathrm{T}_{0}$ : Pulpa de totumo ( M1-totumo verde) sin ensilado, estrujada y sin secado.

$T_{1}$ : Pulpa de totumo en estado M1, ensilado por 4 días, estrujada y secada al sol.

$\mathrm{T}_{2}$ : Pulpa de totumo en estado $M 1$, ensilado por 8 días, estrujada y secada en horno.

$\mathrm{T}_{3}$ : Pulpa de totumo ( M2-totumo maduro) sin ensilado, estrujada y sin secado.

$\mathrm{T}_{4}$ : Pulpa de totumo en estado $\mathrm{M} 2$, ensilada por 4 días, estrujada y secada al sol.

$\mathrm{T}_{5}$ : Pulpa de totumo en estado $\mathrm{M} 2$, ensilada por 8 días, estrujada y secada en horno.

Sehizoel muestreodelostotumosverdesymaduros con cuatro y seis meses de proceso de maduración fisiológica en el árbol, respectivamente. Con base a los tratamientos planteados en el diseño experimental y por medio de los métodos de la Asociación oficial de química analítica AOAC de 1990 se determinó el potencial nutritivo (proteínas, fibra cruda, y carbohidratos), también se evaluaron los parámetros fisicoquímicos $(\mathrm{pH}$, obrix, acidez, índice de madurez, humedad, ceniza) y finalmente los minerales (sodio, potasio, calcio, fósforo y magnesio).

El secado de la pulpa se hizo por radiación solar en láminas de Zinc a una temperatura promedio de 40 grados centígrados y al horno en latas por convección natural a 80 grados centígrados; esto con el objetivo de determinar la influencia de la temperatura en la concentración del ácido cianhídrico. La cantidad del ácido cianhídrico $(\mathrm{HCN})$, se determinó en pulpa seca a $12{ }^{\circ} \mathrm{C}$ por el método de hidrólisis enzimática, aplicada en los laboratorios de Centro Internacional de Agricultura Tropical CIAT en Palmira (Colombia).

\section{RESULTADOS Y DISCUSIÓN}

\section{Resultados fisicoquímicos de la pulpa de totumo en los dos estados de maduración (Verde M1 y Maduro M2).}

La tabla 1 contiene el peso promedio, el rendimiento de la pulpa y la corteza del totumo en los tratamientos respectivos. Se observa que el peso promedio del totumo en el estado de maduración $\mathrm{M} 1$, al inicio del proceso, fue de $721 \mathrm{~g}$ y al final de 658,5 g; el rendimiento de la pulpa antes del proceso del secado en el $\mathrm{T}_{0}$ fue mayor en 4,28\% que en el $\mathrm{T}_{2}$, lo cual es debido a que durante el proceso de ensilaje ocurre deshidratación y una pérdida de peso de $8,60 \%$. El peso del fruto y el rendimiento promedio de la pulpa de todo el proceso de ensilado en el estado de maduración verde fue de 688,20 g y $59,63 \%$ respectivamente.

El peso promedio inicial del totumo maduro, fue de 475,70 g y al final del ensilado de 422,77 g; el mayor rendimiento en pulpa se presentó en $\mathrm{T}_{3}$, con $3,58 \%$ por encima del $\mathrm{T}_{5}$ porque éste último estuvo en un mayor estado de madurez; además durante el ensilado ocurrió una deshidratación y por ende una pérdida de $11,12 \%$ de peso del fruto. Al final de todo el proceso de ensilado del totumo maduro, el peso 
Tabla 1. El Peso, el Rendimiento de pulpa y corteza del totumo en los tratamientos.

\begin{tabular}{cccccc}
\hline & \multirow{2}{*}{$\begin{array}{c}\text { Peso inicial } \\
\text { del totumo }(\mathbf{g})\end{array}$} & \multicolumn{3}{c}{ Pulpa } & \multicolumn{3}{c}{ Condimientos } \\
\cline { 3 - 6 } & & $\mathbf{g}$ & $\mathbf{\%}$ & $\mathbf{g}$ & $\mathbf{\%}$ \\
\hline $\mathrm{T}_{0}$ & 721,00 & 457,29 & 63,42 & 263,66 & 36,57 \\
$\mathrm{~T}_{1}$ & 685,12 & 386,06 & 56,35 & 299,06 & 43,64 \\
$\mathrm{~T}_{2}$ & 658,50 & 389,50 & 59,14 & 269,00 & 40,85 \\
$\mathrm{~T}_{3}$ & 475,70 & 288,19 & 60,58 & 187,50 & 39,41 \\
$\mathrm{~T}_{4}$ & 519,80 & 291,25 & 56,03 & 228,55 & 43,96 \\
$\mathrm{~T}_{5}$ & 422,77 & 240,99 & 57,00 & 181,78 & 42,99 \\
\hline
\end{tabular}

$\mathrm{T}_{0}$ : Pulpa de totumo verde sin ensilado, estrujada y sin secado; $\mathrm{T}_{1}$ : Pulpa de totumo verde, ensilado por 4 días, estrujada y secada al sol; $\mathrm{T}_{2}$ : Pulpa de totumo verde, ensilado por 8 días, estrujada y secada en horno; $\mathrm{T}_{3}$ : Pulpa de totumo maduro sin ensilado, estrujada y $\sin$ secado; $\mathrm{T}_{4}$ : Pulpa de totumo maduro, ensilada por 4 días, estrujada y secada al sol; $\mathrm{T}_{5}:$ Pulpa de totumo maduro, ensilada por 8 días, estrujada y secada en horno.

del fruto y el rendimiento de la pulpa promedios fueron de 472,75 g y 57,87\% respectivamente. Estos resultados son muy parecidos a los reportados por Roncallo et al. (1996).

En la tabla 2 se presenta el comportamiento de las variables humedad, grados brix y acidez del totumo en los tratamientos respectivos.

El $\mathrm{T}_{0}$ que representó la pulpa de totumo en estado de maduración M1 estrujada, sin ensilado y sin secado presentó un $60,12 \%$ de humedad por debajo del arrojado por Figueroa y Bressani 2000. El T 3 que representó la pulpa de totumo en estado de maduración M2 estrujada, sin ensilado y sin secado presentó un $61,79 \%$ de humedad, resultado que se encuentre por debajo del dato arrojado por la investigación (Figueroa y Bressani 2000).

Los grados brix de la pulpa del totumo en todos sus tratamientos aumentaron cuando se iba avanzando en la investigación, arrojando 10 y 14,6 grados como el menor y mayor valor correspondientes a los tratamientos $T_{0} y$ $\mathrm{T}_{4}$ respectivamente. Esto se presentó porque a medida que maduraba el totumo los ácidos probablemente fueron convertidos en azucares.

La acidez en los tratamientos del totumo verde y totumo maduro, manifestaron una tendencia a la reducción de la concentración en porcentaje, desde 0,33 hasta 0,29 y 0,54 hasta 0,40, respectivamente. Este comportamiento se presentó por el mismo proceso de maduración del fruto; sin embargo se notó que el $\mathrm{T}_{2} \mathrm{y}_{3}$ presentaron un leve

Tabla 2. El porcentaje de humedad, grados brix y acidez del totumo en los tratamientos.

\begin{tabular}{lrrrrrr}
\hline \multicolumn{1}{c}{ Variables } & $\mathbf{T}_{\mathbf{0}}$ & $\mathbf{T}_{\mathbf{1}}$ & $\mathbf{T}_{\mathbf{2}}$ & $\mathbf{T}_{\mathbf{3}}$ & $\mathbf{T}_{\mathbf{4}}$ & $\mathbf{T}_{\mathbf{5}}$ \\
\hline Humedad (\%) & 60,12 & 12,50 & 12,90 & 61,79 & 12,7 & 12,80 \\
${ }^{0}$ Brix (\%) & 10,00 & 11,00 & 12,33 & 13,00 & 14,6 & 14,00 \\
Acidez (\%) & 0,33 & 0,23 & 0,29 & 0,54 & 0,40 & 0,40 \\
\hline
\end{tabular}

$\mathrm{T}_{0}$ : Pulpa de totumo verde sin ensilado, estrujada y sin secado; $\mathrm{T}_{1}$ : Pulpa de totumo verde, ensilado por 4 días, estrujada y secada al sol; $\mathrm{T}_{2}$ : Pulpa de totumo verde, ensilado por 8 días, estrujada y secada en horno; $\mathrm{T}_{3}$ : Pulpa de totumo maduro sin ensilado, estrujada y $\sin$ secado; $\mathrm{T}_{4}$ : Pulpa de totumo maduro, ensilada por 4 días, estrujada y secada al sol; $\mathrm{T}_{5}: \mathrm{Pulpa}$ de totumo maduro, ensilada por 8 días, estrujada y secada en horno. 
aumento, esto debido posiblemente a la carencia de homogeneidad y a la mayor liberación de ácido cianhídrico, de la pulpa estrujada.

Los valores promedios de $\mathrm{pH}$ e índice de madurez, se presentan en la tabla 3, donde el análisis de varianza reveló diferencias significativas para el $\mathrm{pH}$, dependiendo del tratamiento. Los tratamientos $\mathrm{T}_{1} \mathrm{yT}_{2}$ presentaron los mayores valores de $\mathrm{pH}$ diferenciándose estadísticamente de los tratamientos $\mathrm{T}_{0^{\prime}}, \mathrm{T}_{3}, \mathrm{~T}_{4}$ y $T_{5}$ los cuales arrojaron los menores valores de $\mathrm{pH}$. Los tratamientos $\mathrm{T}_{0^{\prime}} \mathrm{T}_{1}$ y $\mathrm{T}_{2^{\prime}}$ y los tratamientos $\mathrm{T}_{3}, \mathrm{~T}_{4}$ y $\mathrm{T}_{5}$ presentaron aumento del pH desde 4,36 hasta 4,59 y 4,25 a 4,41 respectivamente, esto se debió, probablemente al proceso de ensilado, al uso de los ácidos orgánicos en la cadena respiratoria y en la conversión de azúcares y a la evaporación de ácidos en el secado de la pulpa (FAO 1999; Wills et al. 1984; Cooke y Maduagwu 1978). El pH es menor en los tres últimos tratamientos (totumo maduro) que en los tres primeros (totumo verde), esto, porque hubo mayor liberación de ácido cianhídrico durante el proceso de estrujado y a la actividad enzimática en la pulpa de totumo maduro (Badui 1993).
El mayor índice de madurez se presentó en el $\mathrm{T}_{1}$ con 47,51 diferenciándose estadísticamente de los demás tratamientos los cuales arrojaron los contenidos más bajos de índice de madurez como consecuencia del menor porcentaje de acidez. De todos los tratamientos el que menor índice de madurez presentó fue $\mathrm{T}_{3}$ con 30,00 debido a que este tratamiento tuvo el mayor porcentaje de acidez. También se observó que el totumo verde inicia con 30,25 de índice de madurez para $T_{1}$ y termina la investigación con un índice de madurez de 42,90 para $\mathrm{T}_{2}$, mientras que el totumo maduro inicia con 30,00 de índice de madurez para $T_{3}$ y termina la investigación con un índice de madurez de 34,09 para $\mathrm{T}_{5}$. Estos resultados se dieron porque los porcentajes de acidez disminuyeron, por la utilización de ácidos orgánicos en la respiración y en la conversión a azúcares. De todos los tratamientos, el $\mathrm{T}_{3}, \mathrm{~T}_{4} \mathrm{y}$ $\mathrm{T}_{5}$ fueron los que presentaron menores índices de madurez, esto debido a que el fruto en el estado de maduración M2, se cosechó con una mayor actividad enzimática la cual produjo un aumento del porcentaje de acidez.

Tabla 3. Las medias de pH, índice de madurez, proteína, carbohidratos, fibra y ácido cianhídrico (HCN) en los tratamientos.

\begin{tabular}{lrrrrrr}
\hline $\begin{array}{l}\text { Variables en } \\
\text { base seca }\end{array}$ & \multicolumn{1}{c}{$\mathbf{T}_{\mathbf{0}}$} & \multicolumn{1}{c}{$\mathbf{T}_{\mathbf{1}}$} & \multicolumn{1}{c}{$\mathbf{T}_{\mathbf{2}}$} & \multicolumn{1}{c}{$\mathbf{T}_{\mathbf{3}}$} & \multicolumn{1}{c}{$\mathbf{T}_{\mathbf{4}}$} & \multicolumn{1}{c}{$\mathbf{T}_{\mathbf{5}}$} \\
\hline $\mathrm{pH}$ & $4,36^{\mathrm{b}}$ & $4,55^{\mathrm{a}}$ & $4,59^{\mathrm{a}}$ & $4,25^{\mathrm{b}}$ & $4,38^{\mathrm{b}}$ & $4,41^{\mathrm{b}}$ \\
Índice madurez & $30,25^{\mathrm{b}}$ & $47,51^{\mathrm{a}}$ & $42,90^{\mathrm{b}}$ & $30,00^{\mathrm{b}}$ & $36,32^{\mathrm{b}}$ & $34,09^{\mathrm{b}}$ \\
Proteína (\%) & $8,44^{\mathrm{b}}$ & $9,96^{\mathrm{a}}$ & $10,32^{\mathrm{a}}$ & $8,84^{\mathrm{b}}$ & $10,29^{\mathrm{a}}$ & $10,56^{\mathrm{a}-{ }^{-}}$ \\
Carbohidrato (\%) & $7,61^{\mathrm{b}}$ & $40,98^{\mathrm{a}}$ & $39,65^{\mathrm{a}}$ & $5,44^{\mathrm{b}}$ & $42,71^{\mathrm{a}}$ & $41,30^{\mathrm{a}}$ \\
Fibra (\%) & $11,43^{\mathrm{b}}$ & $14,26^{\mathrm{a}}$ & $14,23^{\mathrm{a}}$ & $9,43^{\mathrm{b}}$ & $11,20^{\mathrm{b}}$ & $11,63^{\mathrm{b}}$ \\
$\mathrm{HCN}(\mathrm{ppm})$ & $11,00^{\mathrm{a}}$ & $6,50^{\mathrm{b}}$ & $5,50^{\mathrm{b}}$ & $4,00^{\mathrm{b}}$ & $4,00^{\mathrm{b}}$ & $2,00^{\mathrm{b}}$ \\
\hline
\end{tabular}

$\mathrm{T}_{0}$ : Pulpa de totumo verde sin ensilado, estrujada y sin secado; $\mathrm{T}_{1}$ : Pulpa de totumo verde, ensilado por 4 días, estrujada y secada al sol; $\mathrm{T}_{2}$ : Pulpa de totumo verde, ensilado por 8 días, estrujada y secada en horno; $\mathrm{T}_{3}$ : Pulpa de totumo maduro sin ensilado, estrujada y sin secado; $\mathrm{T}_{4}$ : Pulpa de totumo maduro, ensilada por 4 días, estrujada y secada al sol; $\mathrm{T}_{5}$ : Pulpa de totumo maduro, ensilada por 8 días, estrujada y secada en horno.

Los valores medios con letras diferentes como superíndice en la misma fila presentaron diferencias significativas $(P<0,05)$. 
Resultados nutricionales de la pulpa de totumo en los dos estados de maduración (Verde M1 y Maduro M2).

En la tabla 3 donde se presentan los resultados nutricionales, se observa que los tratamientos $T_{1}$, $\mathrm{T}_{4^{\prime}} \mathrm{T}_{2}$ y $\mathrm{T}_{5}$ arrojaron las mayores concentraciones de proteína diferenciándose estadísticamente de los tratamientos $T_{0}$ y $T_{3}$ los cuales presentaron los contenidos de proteína más bajos, debido a que las condiciones del ensilado favorecieron el proceso de maduración del totumo o la síntesis de algunos compuestos proteicos (Wills et al. 1984). Los tratamientos $T_{3^{\prime}} T_{4}$ y $T_{5}$ correspondientes a la pulpa de totumo maduro, presentaron los mayores porcentajes de proteínas; en su orden respectivo 8,84; 10,29; y 10,56; mientras que los tratamientos $T_{0^{\prime}} T_{1}$ y $T_{2}$ de la pulpa de totumo verde, arrojaron en promedio menor concentración proteica; en su orden respectivo 8,44; 9,96 y 10,32. Estos valores se encuentran en el rango de proteína hallado por Nwosu (2008) y menores en los hallado en la pulpa de la Crescentia alata por (Figueroa y Bressani 2000).

Los mayores porcentajes de carbohidrato se presentaron en los tratamientos $\mathrm{T}_{4}, \mathrm{~T}_{5}, \mathrm{~T}_{1} \mathrm{y}_{2}$ con 42,$71 ; 41,30 ; 40,99$ y 39,65 respectivamente, diferenciándose estadísticamente de los tratamientos $\mathrm{T}_{0}$ y $\mathrm{T}_{3}$ los cuales obtuvieron los menores contenidos. Los valores mas altos de los tratamientos se dieron porque durante el proceso de ensilado hubo una maduración (conversión de ácidos en azucares) y al secado donde los carbohidratos se concentraron. Estos resultados son menores a los arrojados por Figueroa y Bressani (2000), hallados en la pulpa de la Crescentia alata; y menores a los encontrados por Nwosu (2008).

Los tratamientos $T_{1}$ y $T_{2}$ presentaron los contenido defibramasaltos diferenciándoseestadísticamente de los demás tratamientos, los cuales obtuvieron los contenidos de fibras más bajos. Los mayores valores de los tratamientos se dieron gracias al efecto positivo que tuvo el proceso de maduración desarrollado en el ensilado y al secado. Estos porcentajes se encuentran por encima de las concentraciones de fibra publicadas por Nwosu (2008). El T 1 presentó mayor porcentaje y el de menor concentración fue $\mathrm{T}_{3}$.

\section{Resultados del ácido cianhídrico presente en la pulpa de totumo en los dos estados de maduración (Verde M1 y Maduro M2).}

La tabla 3 presenta las concentraciones de ácido cianhídrico hallados en la pulpa de totumo. Todos los tratamientos arrojaron resultados de $\mathrm{HCN}$ por debajo de las concentraciones permitidas para la alimentación animal, publicadas por el diario de la comunidad europea (1999). Los valores o ppm de Acido Cianhídrico hallados por los investigadores Inuyama y Kaji (1969) y Nwosu (2008), con ensilajes de sorgo y calabaza respectivamente, están muy por encima de los encontrados en esta investigación; donde el tratamiento $\mathrm{T}_{0}$ presentó el mayor contenido de $\mathrm{HCN}$ con 11 ppm siendo significativamente diferente al resto de los tratamientos.

\section{Resultados de los minerales de la pulpa de totumo en los dos estados de maduración (Verde M1 y Maduro M2).}

En la tabla 4 se muestran los valores promedios de las concentraciones de ceniza y minerales en la pulpa de totumo de los diferentes tratamientos.

Los tratamientos $\mathrm{T}_{3} \quad$ y $\mathrm{T}_{5}$ presentaron los mayores porcentajes de ceniza diferenciándose estadísticamente del resto. El tratamiento $T_{2}$ fue el que tuvo menos porcentaje de ceniza. 
Tabla 4. La Composición mineral de la pulpa del totumo para los diferentes tratamientos.

\begin{tabular}{|c|c|c|c|c|c|c|}
\hline $\begin{array}{l}\text { Variables en } \\
\text { base seca }\end{array}$ & $\mathbf{T}_{0}$ & $\mathbf{T}_{1}$ & $\mathbf{T}_{2}$ & $\mathbf{T}_{3}$ & $\mathrm{~T}_{4}$ & $\mathbf{T}_{5}$ \\
\hline Ceniza (\%) & $5,80^{b}$ & $6,30^{\mathrm{b}}$ & $6,00^{b}$ & $7,60^{\mathrm{a}}$ & $6,10^{b}$ & $6,80^{\mathrm{a}}$ \\
\hline Sodio (mg/100 g) & $1,39^{\mathrm{a}}$ & $1,02^{\mathrm{b}}$ & $0,90^{\mathrm{b}}$ & $1,29^{\mathrm{a}}$ & $1,08^{b}$ & $0,94^{b}$ \\
\hline Potasio ((mg/100 g) & $0,47^{\mathrm{a}}$ & $0,19^{b}$ & $0,13^{b}$ & $0,40^{\mathrm{a}}$ & $0,20^{b}$ & $0,15^{b}$ \\
\hline Calcio ((mg/100 g) & $0,32^{\mathrm{a}}$ & $0,21^{b}$ & $0,17^{b}$ & $0,38^{a}$ & $0,25^{b}$ & $0,18^{b}$ \\
\hline Fósforo ((mg/100 g) & $0,15^{\mathrm{a}}$ & $0,10^{\mathrm{b}}$ & $0,07^{b}$ & $0,18^{\mathrm{a}}$ & $0,11^{b}$ & $0,08^{\mathrm{b}}$ \\
\hline Magnesio (mg/100 g) & $0,19^{a}$ & $0,12^{\mathrm{b}}$ & $0,09^{b}$ & $0,23^{a}$ & $0,17^{b}$ & $0,13^{b}$ \\
\hline
\end{tabular}

$\mathrm{T}_{0}$ : Pulpa de totumo verde sin ensilado, estrujada y sin secado; $\mathrm{T}_{1}$ : Pulpa de totumo verde, ensilado por 4 días, estrujada y secada al sol; $T_{2}$ : Pulpa de totumo verde, ensilado por 8 días, estrujada y secada en horno; $T_{3}$ : Pulpa de totumo maduro sin ensilado, estrujada y sin secado; $\mathrm{T}_{4}$ : Pulpa de totumo maduro, ensilada por 4 días, estrujada y secada al sol; $\mathrm{T}_{5}$ : Pulpa de totumo maduro, ensilada por 8 días, estrujada y secada en horno.

Los valores de las medias en la misma línea con letras diferentes como superíndice presentaron diferencias significativas $(\mathrm{P}<0,05)$.

Esto se presentó porque el proceso de secado al horno y calcinación en mufla afectó negativamente la concentración de la ceniza. Estas concentraciones de ceniza son mayores que las concentraciones arrojadas en la pulpa de la calabaza según Nwosu (2008); pero muy cercanas a las encontradas por Gómez-Brenes (1980) en la pulpa del morro o Crescentia alata.

El análisis de varianza reveló efecto significativo del $\mathrm{Na}$ según el tratamiento. $\mathrm{El}_{0} \mathrm{~T}_{0} \mathrm{~T}_{3}$ fueron los que obtuvieron mayor cantidad de $\mathrm{Na}$, diferenciándose estadísticamente del resto de los tratamientos. Esto muestra que los procesos de ensilado y secado provocaron una disminución en el contenido de sodio. La mayor concentración (1,39 mg / 100 g) de este mineral se presentó en el $\mathrm{T}_{0}$ y la menor $(0,9 \mathrm{mg} / 100 \mathrm{~g})$ en el $\mathrm{T}_{2}$. Sin embargo, todos estos resultados se encuentran por debajo de las concentraciones de sodio halladas en pulpa de calabaza por Nwosu (2008).

Los tratamientos que no se ensilaron ni se secaron, $\mathrm{T}_{0}$ y $\mathrm{T}_{3}$, fueron los que presentaron mayor contenido de potasio, calcio, fosforo $y$ magnesio diferenciándose estadísticamente de los demás tratamientos (tabla 4). El de menor contenido de estos minerales se encontró en el $\mathrm{T}_{2^{\prime}}$ debido a que el secado de la pulpa al horno y calcinado en la mufla afectó de forma negativa las concentraciones de dichos elementos. Hubo en general mayores promedios de concentraciones de minerales en la pulpa de totumo maduro, que en la pulpa de totumo verde. Sin embargo, estos valores son menores en comparación con los datos publicados por Nwosu (2008).

\section{CONCLUSIONES}

El proceso de ensilado, estrujado y secado de la pulpa de totumo en sus dos estados de maduración arrojó resultados positivos, ya que se determinaron pocas concentraciones de HCN. Desde el punto de vista nutricional la pulpa del totumo maduro, con ocho días de ensilado, estrujada y secada al horno se considera como la alternativa más conveniente para alimentación bovina por presentar los mayores contenidos de proteínas y minerales. 


\section{REFERENCIAS}

Asociación oficial de química analítica (AOAC), métodos oficiales de análisis, Editado por S. Williams. Publicado en 1990 por la AOAC, Inc. USA p1015.

Badui, J. 1993. Química de los alimentos, Editorial Alambra, México. p 60-65.

Cooke, R.D. y Maduagwu, E.N. 1978. LOS efectos de cianuro contenido en la elaboración sencilla de trozos de yuca. J. Food Technol 13: p 299-306. Tomado del seminario de Ottawa Canadá en 1982.

\section{Diario Oficial de las Comunidades Europeas}

4. 5. 1999, Directiva 1999/29/CE DEL Consejo de 22 de abril de 1999 relativa a las sustancias y productos indeseables en la alimentación animal.

FAO1999. Uso del ensilajeen el trópico privilegiado opciones para pequeños campesinos. Tomada de las memorias de la Conferencia Electrónica de la FAO sobre el Ensilaje en los Trópicos, Roma 1 de septiembre a 15 diciembre de 2001, p60-70.

Figueroa, S. y Bressani, R. 2000. Recursos alimenticios vegetales con potencial de explotación agroindustrial. Elaboración de leche vegetal a partir de la semilla del fruto de morro (Crescentia alata L). Reportado por archivos latinoamericanos de nutrición, Universidad del valle, Guatemala p164-170.

Gómez-Brenes, R. A. 1980. Evaluación biológica de harina de morro o jicaro (Crescentia alata L) preparada por ensilaje y / o deshidratación. Institución de Nutrición de Centroamérica y panamá, Archivos Latinoamericanos de Nutrición, Venezuela 30(4):517-538.

Inuyama, S. y M. Kaji. 1969. Concentración del contenido del ácido cianhídrico en diferentes partes del sorgo forrajero. J. Jap. Soc. Grsld. Sci. Herbage Abst 43(2), 10.

Murgueitio, E. y Ibrahim, M. 2004. Conferencia Agroforestería, Ganadería y medio ambiente en américa latina, XII congreso venezolano de producción e industria animal p190-192.

Nwosu, M. 2008. La composición nutritiva y antinutritiva de la pulpa del fruto de la calabaza (Crescentia cujete L) Nigeria. Consultada en febrero del 2010 en el Diario de tecnología de alimentos, Medwell online. Revista de tecnología de los alimentos 6(6):267-270.

Roncallo, B., Navas, A. y Caribella, A. 1996. Potencial de los frutos de plantas nativas en la alimentación de rumiantes, sistema silvopastoriles, alternativas para la ganadería. Valledupar, Colombia p81-92.

Wills, R., Lee, T., Mc, Glasson. B. y Graham, D. 1984. Fisiología y Manipulación de Frutas y Hortalizas Post-Recolección. Zaragoza, España. Acribia p18-22, 43-46, 81-83.

Zamora, S., García, J., Bonilla, G., Aguilar, H., Harvey, C. y Ibrahim, M. 2001. Cómo utilizar los frutos de guanacaste (Enterolobium cyclocarpum), guácimo (Guazuma ulmifolia), genízaro (Pithecellobiug saman) y jícaro (Crescentia alata) en alimentación animal? Agrofestería en las Américas 8(31):45-49. 